\title{
REAL FUNCTIONS COMING FROM FLOWS ON COMPACT SPACES AND CONCEPTS OF ALMOST PERIODICITY
}

\author{
BY \\ L. AUSLANDER(1) AND F. HAHN
}

Introduction. 1. A continuous real valued function $f$ of the reals $T$ is said to be uniformly almost periodic [3] if for each $\varepsilon>0$ there is a relatively dense subset $S \subset T$ such that

$$
|f(t+s)-f(t)|<\varepsilon
$$

for $t \in T$ and $s \in S$. This definition can be restated in equivalent ways in terms of particular flows. A flow $(X, T)$ is a transformation group [8] where $X$ is a topological space and $T$ is the additive group of the reals. We designate the action of $t \in T$ on $x \in X$ by $x \rightarrow x_{t}$. A point $x \in X$ is said to be almost periodic if for each neighborhood $U$ of $x$ there is a relatively dense set $S \subset T$ such that $x_{s} \in U$ if $s \in S$. We let $B$ be the Banach space of uniformly continuous bounded real valued functions of the reals. The norm of an element of $B$ shall be the supremum of its absolute values. We let $(B, T)$ be the flow defined by $x \rightarrow x_{t}$ where $x_{t}(s)$ $=x(t+s)$. Uniformly almost periodic functions may be defined in any one of the three following ways.

(1) A function $x$ in $B$ is uniformly almost periodic if $x$ is an almost periodic point of $(B, T)$.

(2) A function $x$ in $B$ is uniformly almost periodic if its orbit closure in $(B, T)$ is compact.

(3) A function $x$ in $B$ is uniformly almost periodic if it may be extended to a continuous function on the Bohr compactification of the reals.

The fact that these three statements are equivalent may be found in $[3 ; 12]$.

For many notions of functions being recursive under translation the compactopen topology is more natural than the topology of uniform convergence. With this in mind we let $C$ be the set of continuous real valued functions of the reals with the compact open topology. This topology is equivalent to the topology induced by the metric

$$
\rho(x, y)=\sup _{0<s}\left\{\min \left\{\sup _{|t|<s}|x(t)-y(t)|, 1 / s\right\}\right\} .
$$

Received by the editors February 15, 1962.

(1) Research supported by NSF 15565 and 18995. 
With this metric $C$ is complete and $(C, T)$ is a flow where $T$ acts by translation. This is also called the dynamical system of Bebutov [10].

The previous definitions suggest that we investigate the following classes of functions.

$1^{\prime}$. Let $W$ be the set of all $x \in C$ whose orbits in $(C, T)$ are almost periodic.

$2^{\prime}$. Let $A(c)$ be the set of all $x \in W$ whose orbit closures in $(C, T)$ are compact.

$3^{\prime}$. Let $A(P)$ be the set of all $x \in C$ which come from a universal minimal set with property $P$. (In the body of the paper $P$ will represent different properties.)

The purpose of this paper is to study these classes of functions. We prove that $W$ properly contains $A(c)$. If $A(M)$ is the class of all functions coming from the universal minimal flow we prove $A(M)=A(c)$. We will give examples to show that neither of the preceding classes are vector spaces. If $A(D)$ is the set of functions coming from the universal distal minimal flow then $A(D)$ is a lattice algebra of functions and is properly contained in $A(M)$. It is known that the set of uniformly almost periodic functions is the same as $A(E)$, where $A(E)$ designates the set of functions coming from the universal equicontinuous minimal set (see Chu [4]). We also show that some functions of $A(M)$ do not have mean values, that is, there is an $x \in A(M)$ for which $\lim _{s \rightarrow+\infty}(1 / 2 s) \int_{-s}^{s} x(t) d t$ does not exist. It is known [3] that the functions of $A(E)$ do have mean values. To the best knowledge of the authors this question is not answered for $A(D)$.

The authors would like to take this opportunity to acknowledge many helpful conversations with G. A. Hedlund.

2. The almost periodic points of $(C, T)$. A point $x$ of $C$ is said to be almost periodic if for each $\varepsilon>0$ there is a relatively dense set $S \subset T$ such that $\rho\left(x, x_{s}\right)<\varepsilon$ if $s \in S$. We let $W$ be the collection of all almost periodic points of $(C, T)$. It is easy to see that $W$ is invariant under $T$. The set $W$ is distinguished by its lack of regularity. To show this we construct some examples of functions in $W$ which illustrate the pathology of this set.

EXAMPLE 2.1. We first show that there exists in $W$ two bounded uniformly continuous functions $x$ and $y$ such that $x+y \notin W$. These functions are obtained by a modification of the Morse bisequence [9]. If $m$ is an integer the values $x(m)$ and $y(m)$ shall be restricted to bring either 1 or zero. If we let $B_{n}$ be a finite sequence of zeros and ones, then $B_{n}^{\prime}$ shall be that sequence obtained from $B_{n}$ by replacing one by zero and zero by one. If $B_{1}$ and $B_{2}$ are two finite sequences then $B_{1} B_{2}=B_{3}$ is the sequence obtained by writing first $B_{1}$ and then $B_{2}$. We now define the Morse bisequence (see also [8, Chapter 12;9]). The definition is inductive and is given as follows

$$
\begin{aligned}
B_{0} & =0 \\
B_{n+1} & =B_{n} B_{n}{ }^{\prime} .
\end{aligned}
$$

We let $B=\bigcup^{\infty}=0 B_{n}$. We define $\bar{B}$ to be the reflection of $B$. The bisequence 
$\{x(n): n$ an integer $\}$ is then defined by $\bar{B} \cdot B$. We also define the bisequence $\{y(n): n$ an integer $\}$ by $\bar{B}^{\prime} \cdot B$ where prime again means interchanging zero and one.

These bisequences are now extended to real valued functions as follows: let $x(n+1 / 2)=y(n+1 / 2)=0$ for integral $n$. Now make the functions linear between the points on which they are already defined. These functions are uniformly continuous since any linear piece has either slope zero or \pm 2 .

It is known that the bisequences $\{x(n): n$ integral $\}$ and $\{y(n): n$ integral $\}$ are almost periodic under the shift transformation [8;9]. We notice that $x(n)+y(n)$ $=1$ for $n$ negative. Since both $x(n+1 / 2)$ and $y(n+1 / 2)$ are zero for each $n$ we see $x(n+1 / 2)+y(n+1 / 2)=0$. On the other hand if $n$ is positive then $x(n)+y(n)=2$ or 0 . In particular $x(1)+y(1)=2$. Since $\rho(x, y)<\varepsilon$ if and only if $\sup _{|t|<1 / \varepsilon}|x(t)-y(t)|<\varepsilon$ we see that $\rho\left(x+y,(x+y)_{t}\right)>1 / 2$ if $t$ is an integer less than -2 . Thus $x+y$ is not almost periodic for integral values of $t$ and consequently is not almost periodic for real values of $t[8$, p. 31$]$.

EXAMPLE 2.2. In this example we give a method for the construction of two functions in $W$. One of these functions is not uniformly continuous, is not bounded and does not have a mean value. The other function is bounded, non-negative, but not uniformly continuous. The construction of both these functions depends on the existence of a certain subset $B$ of the reals.

The idea of this construction is not too difficult but the details are laborious. A brief outline of the idea is in order. We begin with a continuous function $f$ defined on a finite symmetric interval about zero and $f$ vanishes at the endpoints. To the original interval adjoin two intervals of length $<1 / 2$ on each end so the new interval is symmetric. We now extend $f$ to the larger interval such that the modulus of continuity is decreased by more than $1 / 2$ and the extension vanishes at the endpoints. The next step is to translate the previous interval to the right and left so we have three adjoining intervals. The function is extended by taking its translates. We now repeat the first step adjoining intervals of length ess than $1 / 2^{2}$. We then repeat the second step. We continue inductively alternating these two procedures. The fact that at each odd step the modulus of continuity is decreased by more than $1 / 2$ says that the function cannot be uniformly continuous. The function is forced to be in $W$ because of the translation of the even numbered sets and the fact that the sum of the lengths of the new intervals added is finite. We choose to use this inductive procedure to describe a discrete subset $B$ of the reals. We then define a polygonal function whose zeros contain $B$.

If $B_{i}$ is a finite subset of the reals let $m_{i}$ be the maximum element of $B_{i}$. Let $B_{0}$ be any finite symmetric subset of the reals which has a nonzero element. Let $B_{1}=\left\{-a_{0}\right\} \cup B_{0} \cup\left\{a_{0}\right\}$ where $0<\left(a_{0}-m_{0}\right)<1$. Suppose we have defined $B_{j}$ for $j<2 n$. Let

$$
B_{2 n}=\left(-2 m_{2 n-1}+B_{2 n-1}\right) \cup B_{2 n-1} \cup\left(2 m_{2 n-1}+B_{2 n-1}\right)
$$


and

$$
B_{2 n+1}=\left\{-a_{n}\right\} \cup B_{2 n} \cup\left\{a_{n}\right\} \text { where } 0<\left(a_{n}-m_{2 n}\right)<1 / 2^{n} .
$$

Let $B=\bigcup_{i=0}^{\infty} B_{i}$ and notice that $\sum_{i=0}^{\infty}\left(a_{i}-m_{2 i}\right)$ converges absolutely.

Proposition 1. B is symmetric.

Proof. $B_{0}$ is symmetric and we see by induction that $B_{i}$ is symmetric for each $i$ and therefore so is $B$.

Proposition 2. Let $s_{n}=\sum_{i=0}^{n}\left(a_{i}-m_{2 i}\right)$. There is a sequence of non negative numbers $b_{n}$ such that $b_{n}+B_{0}$ and $-b_{n}+B_{0}$ is contained in $B_{2 n}$ and in $B_{2 n+1}$ and $m_{2 n}-\left(b_{n}+m_{0}\right)<s_{n}$ and $m_{2 n+1}-\left(b_{n}+m_{0}\right)<s_{n}$.

Proof. We prove this by induction on $n$. If $n=0$ we choose $b_{0}=0$ and our result holds. Suppose the result is true for $n \leqq p$. Since $B_{2 p+2}=\left(-2 m_{2 p+1}\right.$ $\left.+B_{2 p+1}\right) \cup B_{2 p+1} \cup\left(2 m_{2 p+1}+B_{2 p+1}\right)$ we need only let $b_{p+1}=b_{p}+2 m_{2 p+1}$ and we see that $m_{2 p+2}-\left(b_{p+1}+m_{0}\right)=3 m_{2 p+1}-\left(b_{p}+2 m_{2 p+1}+m_{0}\right)<s_{p}$ $<s_{p+1}$. Since $B_{2 p+3}=\left\{-a_{p+1}\right\} \cup B_{2 p+2} \cup\left\{a_{p+1}\right\}$ we see that $m_{2 p+3}-\left(b_{p+1}\right.$ $\left.+m_{0}\right)<s_{p}+a_{p+1}<s_{p+1}$. This concludes the proof.

Proposition 3. There is a relatively dense discrete set $S$ such that if $s \in S$ then $s+B_{0} \subset B$, and if $s$ and $t \in S$ such that $s \neq t$ then $s+B_{0} \cap t+B_{0}=\varnothing$.

Proof. We will construct the set $S$ by induction. We wish to define an increasing sequence of sets $S_{i}$ having the following properties:

1. $S_{i}$ is discrete and symmetric.

2. For each $i$ the number $b_{i}$ of Proposition 2 is the greatest element of $S_{2 i}$ and $S_{2 i+1}$.

3. If $s \in S_{i}$ then $s+B_{0} \subset B_{2 i} \subset B_{2 i+1}$.

4. If $s$ and $t \in S_{i}, s \neq t$, then $s+B_{0} \cap t+B_{0}=\emptyset$.

5. If $s$ and $t \in S_{2 i}$ or $S_{2 i+1}$ are adjacent elements then the distance between $s+B_{0}$ and $t+B_{0}$ is not more than $2 s_{i-1}+2 m_{0}$.

Clearly if we let $S=\bigcup_{i=0}^{\infty} S_{i}$ then $S$ satisfies the theorem. We proceed with the construction of the $S_{i}$ 's.

Let $S_{0}=S_{1}=\{0\}$. Suppose $S$ is defined satisfying 1 thru 5 for $n<2 p$. Let $S_{2 p}=\left\{-2 m_{2 p-1}+S_{2 p-1}\right\} \cup S_{2 p-1} \cup\left\{2 m_{2 p-1}+S_{2 p-1}\right\}$ and $S_{2 p+1}=S_{2 p}$, where $m_{2 p-1}$ is the maximum element of $B_{2 p-1}$. If is clear that 1 holds for $S_{2}$ and $S_{2 p+1}$. Since $b_{p}=b_{p-1}+2 m_{2 p-1}$ we see that 2 holds for $S_{2 p}$ and $S_{2 p+1}$. Since

$$
\begin{aligned}
B_{2 p} & =\left\{-2 m_{2 p-1}+B_{2 p-1}\right\} \cup B_{2 p-1} \cup\left\{2 m_{2 p-1}+B_{2 p-1}\right\}, \\
B_{2 p+1} & =\left\{-a_{p}\right\} \cup B_{2 p} \cup\left\{a_{p}\right\},
\end{aligned}
$$

it follows that 3 holds.

Proposition 2 says that $m_{2 p-1}-\left(b_{p-1}-m_{0}\right)<s_{p-1}$, therefore $2 m_{2 p-1}$ 
$-2 b_{p-1}<2 s_{p-1}+2 m_{0}$. Since the largest element of $S_{2 p-1}$ is $b_{p-1}$ and the smallest element of $2 m_{2 p-1}+S_{2 p-1}$ is $2 m_{2 p-1}-b_{p-1}$ we see that 5 holds. We see inductively that $\left(2 m_{2 p-1}-b_{p-1}\right)-b_{p-1}=2\left(m_{2 p-1}-b_{p-1}\right)>2 m_{0}$. From this the validity of 4 follows.

Proposition 4. For each $n$ there is a relatively dense discrete set $S_{n}$ such that if $s \in S_{n}$ then $s+B_{2 n} \subset B$ and if $s$ and $t \in S_{n}$ such that $s \neq t$ then $s+B_{2 n} \cap t+B_{2 n}=\varnothing$.

Proof. We let $A_{0}=B_{2 n}$ and $A_{1}=\left\{-a_{n}\right\} \cup B_{2 n} \cup\left\{a_{n}\right\}$. In general we let $A_{2 j}=\left\{2 M_{2 j-1}+A_{2 j-1}\right\} \cup A_{2 j-1} \cup\left\{2 M_{2 j-1}+A_{2 j-1}\right\} A_{2 j+1}=\left\{-a_{j n}\right\}$ $\cup A_{2 j} \cup\left\{a_{j n}\right\}$, where $M_{j}$ is the maximum element of $A_{j}$. We have thus made $B_{2 n}=A_{0}$ play the role of $B_{0}$ and Propositions 1, 2, 3 give our result.

Suppose now we have the set $B$. We let $\alpha_{n}$ be any sequence of numbers and let $I_{n}$ be the closed interval spanned by $B_{n}$. We define a function $x$ as follows.

Let $x(t)=0 \quad t \in I_{0}$.

This defines $x(t)$ on $I_{0}$. Let $c_{0}=\left(m_{0}+a_{0}\right) / 2$ and on $I_{1}$ we extend $x$ from $I_{0}$ to $I_{1}$ such that $x$ is symmetric, $x\left(c_{0}\right)=\alpha_{0}, x\left(a_{0}\right)=0$ and the graph of $x$ is linear on the remainder of $I_{1}$.

Suppose $x$ has been defined on $I_{2 p-1}$ such that $x$ vanishes at the endpoints of the interval. Since $B_{2 p}=-\left\{2 m_{2 p-1}+B_{2 p-1}\right\} \cup B_{2 p-1} \cup\left\{2 m_{2 p-1}+B_{2 p-1}\right\}$ we extend $x$ to $I_{2 p}$ by translation to the right and left of the function $x$ on $I_{2 p-1}$. Let $c_{p}=\left(m_{p}+a_{p}\right) / 2$ and on $I_{2 p+1}$ extend $x$ from $I_{2 p}$ to $I_{2 p+1}$ such that $x$ is symmetric, $x\left(c_{p}\right)=\alpha_{p}, x\left(a_{p}\right)=0$ and the graph of $x$ is linear on the remainder of $I_{2 p+1}$. The function $x$ is then defined on the reals and Propositions 1, 2, 3, 4 tell us that $x$ is an almost periodic point of $(C, T)$.

By induction we easily estimate the following integrals

$$
\begin{aligned}
\int_{I_{0}} x(t) d t & =0, \\
\int_{I_{1}} x(t) d t & =\alpha_{0}\left(a_{0}-m_{0}\right), \\
\int_{I_{2 n}} x(t) d t & =\sum_{i=0}^{n-1} 3^{n-i} \alpha_{i}\left(a_{i}-m_{i}\right), \\
\int_{I_{2 n+1}} x(t) d t & =\sum_{i=0}^{n} 3^{n-i} \alpha_{i}\left(a_{i}-m_{i}\right)
\end{aligned}
$$

for $n>0$.

It is simple enough to pick the $\alpha_{i}$ such that $\left(1 / 2 m_{n}\right) \int_{I_{n}} x(t) d t \rightarrow+\infty$ as $n \rightarrow+\infty$. This shows that $x(t)$ is neither uniformly continuous, bounded, nor does it have a mean value.

If we let the $\alpha_{i}=1$ for all $i$ then $x(t)$ is bounded, non-negative and not uniformly continuous. 
3. The totally bounded orbits of $(C, T)$. Since $C$ is a complete metric space the orbit of a point $x$ in $C$ is totally bounded if and only if its orbit closure is compact. We shall make use of the following theorem.

THEOREM 3.1. The orbit closure of $x$ in $C$ is compact if and only if

1. $x$ is uniformly continuous and,

2. $x$ is bounded.

Proof. [8, p. 10, 1.65].

COROllary 3.2. There exist almost periodic points of $(C, T)$ whose orbit closures are not compact.

Proof. In Example 2 we constructed an almost periodic point $x$ of $(C, T)$ which was not uniformly continuous. Apply Theorem 3.1.

We also see that if $x(t)=\operatorname{arccot} t$ (taking the principle values only) then the orbit of $x$ has compact closure. However, $x$ is asymptotic to 0 in the negative direction and is asymptotic to $\pi$ in the positive direction. Thus we see that there exist functions with compact orbit closures in $(C, T)$ which are not in $W$.

We also point out that there are functions whose orbit closures in $(C, T)$ are compact but which are not almost periodic in the sense of Eberlein [7]. The function $x(t)=\operatorname{arccot} t$ is such a function. Let us identify $x$ with its extension to the Stone-Cech compactification $\beta(T)$ of the reals. There is a point $s \in \beta(T)$ such that $x_{t}(s)=\pi$ for each $t$ in $T$. Let $f$ be the linear functional defined on $B$ as follows. $f(y)=y(s)$ for $y \in B$. Thus we see that $f\left(x_{t}\right)=\pi$ for all $t \in T$. Let $g$ be the linear functional defined on $B$ by $g(y)=y(0)$ for each $y \in B$. As $n \rightarrow+\infty$ we see that $g\left(x_{-n}\right) \rightarrow 0$ and $f\left(x_{-n}\right) \rightarrow \pi$. Consequently $x_{-n}$ can have no subset which converges weakly. Thus the orbit of $x$ is not precompact in the weak topology. This is the negation of almost periodicity in the sense of Eberlein.

Since we are predominantly interested in recursive properties we make the following definition.

Definition 3.3. Let $A(c)$ be the set of all functions in $W$ whose orbit closures in $(C, T)$ are compact.

We see that $A(c)$ is a subset of $W$ and we have already remarked that this inclusion is proper.

In number 3 of the Introduction we defined a uniformly almost periodic function in terms of the Bohr compactification of the reals. We will show here that the set $A(c)$ can be described in terms of the universal minimal set.

The Bohr compactification of the reals may be looked upon as being the largest compact solenoidal group. That is, $L(T)$ (the Bohr compactification of $T$ ) is a compact group which has a dense one parameter subgroup and if $G$ is any other compact group with a dense one parameter subgroup then there is a homomorphism $\pi$ of $L(T)$ onto $G$ (see [4]). We generalize this as follows: A flow $(X, T)$ 
is said to be minimal if each orbit is dense in $X$. A compact minimal flow $(M, T)$ is called universal if $M$ is compact and if whenever $(X, T)$ is a compact minimal flow then there is a continuous mapping $\pi$ of $M$ onto $X$, which commutes with the action of $T$. Such a mapping $\pi$ will be called a homomorphism $\left({ }^{2}\right)$.

The following theorem (see Chu [4]) is the analogue to the existence and uniqueness of the Bohr compactification.

THEOREM 3.4. There is a unique compact universal minimal flow.

We will say that a function $x \in C$ comes from a compact minimal flow $(X, T)$ if there is a point $p \in X$, and a continuous real valued function $f$ with domain $X$ such that $f\left(p_{t}\right)=x(t)$ for all $t \in T$.

Definition 3.5. We let $A(M)$ be the set of all functions of $C$ which come from the universal minimal flow $(M, T)$.

LemMa 3.6. If a function $x$ of $C$ comes from any minimal flow $(X, T)$ then it is in $A(M)$.

Proof. Let $f: X \rightarrow T$ and $p \in X$ such that $f\left(p_{t}\right)=x(t)$. Let $\pi$ be a homomorphism of $M$ onto $X$ and let $q \in \pi^{-1}(p)$. It follows that $x(t)=f\left(p_{t}\right)=f \circ \pi\left(q_{t}\right)$.

THEOREM 3.7. $A(M)=A(c)$.

Proof. Let $x \in A(c)$. Since the orbit closure of $x$ in $(C, T)$ is compact and $x$ is an almost periodic point it follows that the orbit closure of $x$ is a compact minimal set $[8$, p. 31]. Let $(Y, T)$ be the flow restricted to this orbit closure. Define $f: Y \rightarrow T$ as follows $f(y)=y(0)$. We see that $x(t)=x_{t}(0)=f\left(x_{t}\right)$. Thus $x \in A(M)$ and $A(c) \subset A(M)$.

Suppose now $x \in A(M), p \in M$ and $f: M \rightarrow T$ such that $f\left(p_{t}\right)=x(t)$. Since $M$ is compact, we will see that $x$ is uniformly continuous. For let $\varepsilon>0$ be given. If we let $v(q, t)=q_{t}$ for $(q, t) \in M \times T$ then we see that $v$ restricted to $M \times[-1,1]$ is uniformly continuous since $M$ is compact. Since $f: M \rightarrow T$ is uniformly continuous it follows that there is a $\delta, 0<\delta<1$, for which $\left|f(q)-f\left(q_{t}\right)\right|<\varepsilon$ for all $q \in M$ and $0 \leqq|t|<\delta$. In particular we have $|x(s+t)-x(s)|=\mid f\left(\left(p_{s}\right)_{t}\right)$ $-f\left(p_{s}\right) \mid<\varepsilon$ whenever $s \in T$ and $0 \leqq|t|<\delta$. The image of $M$ under $f$ is compact so $x(T)$ has compact closure. It follows from Theorem 3.1 that the orbit closure of $x$ is compact. We must now prove $x \in W$.

Let $I$ be any compact interval of real numbers containing zero and let $\varepsilon>0$ be arbitrary. Since $f$ is uniformly continuous and $(M, T)$ is continuous in both variables there is a neighborhood $V$ of $p$ in $M$ such that if $q \in V$ then $\left|f\left(p_{t}\right)-f\left(q_{t}\right)\right|$ $<\varepsilon$ for $t \in I$. Since $(M, T)$ is a compact minimal flow the orbit of $p$ is almost periodic and thus there is a relatively dense set $S=\{s\}$ such that $x_{s} \in V$ for $s \in S$. From this we conclude that $x \in W$ and thus $A(M) \subset A(c)$.

(2) Since there is little chance for confusion we use the phrase "a homomorphism of $X$ onto $Y$ " instead of the customary "a homomorphism of $(X, T)$ onto $(Y, T)$." 
The set $A(M)$ again has the lack of structure noticed about $W$. The two functions $x$ and $y$ of Example 2.1 are in $W$ and have compact orbit closure. Thus they are in $A(M)$. Their sum $x+y$ is not in $W$ and thus not in $A(M)$.

The fact that the functions of $A(M)$ need not have a mean value was first shown by Markov. Here we modify Oxtoby's construction. In [11, p. 134] Oxtoby constructs a compact set $X$ which is minimal under an action of the integers $I$. He also finds a point $p \in x$ and a continuous non-negative function $f: X \rightarrow T$ such that if $x(n)=f\left(p_{n}\right)$ then $x$ does not have a mean value over the integers. We now generalize this example by using the standard technique of embedding $(X, I)$ in a flow $(Y, T)$. We let $Y=X \times[0,1]$ with the following identification $(q, 1)=\left(q_{1}, 0\right)$. The compact flow $(Y, T)$ is defined by $(q, s)_{t}=(q, s+t)$. We notice that since $(X, I)$ is minimal so is $(Y, T)$.

We now define $g: Y \rightarrow T$ as follows $g(q, s)=f(q)\left(1-4(s-1 / 2)^{2}\right),(q, s) \in Y$. Noticing that $g(q, 1 / 2)=f(q)$ we see that $\lim _{n \rightarrow \infty}(1 / 2 n) \int_{-n}^{n} g\left((p, 1 / 2)_{t}\right) d t \geqq(1 / 2)$ $\lim (1 / 2 n) \sum_{i=-n}^{n} f\left(p_{i}\right)$. Since $f\left(p_{i}\right)=x(i)$ does not have a mean value over the integers it follows that $y(t)=g\left((p, 1 / 2)_{t}\right)$ does not have a mean value over the reals.

4. Functions which come from distal transformation groups. The uniformly almost periodic functions form an invariant subalgebra of $C$. We have seen that the invariant subsets $W$ and $A(M)$ are not algebras. Our desire is to find invariant subsets of $W$ which are algebras and are not contained in the set of uniformly almost periodic functions. These thoughts bring our attention to distal flows as possible generators of such algebras.

Definition 4.1. A flow $(X, T)$ is called distal if for any three points $p, q, r \in X$ and any net $\left\{t_{n}: n \in D\right\} \subset T$ for which $t_{n} p \rightarrow r$ and $t_{n} q \rightarrow r$ then $p=q$.

If $\left(X_{\alpha}, T\right), \alpha \in A$, is a family of flows we define the product flow

$$
\left(\prod_{a \in a} X_{\alpha}, T\right)
$$

as follows: if $x=\left(x_{\alpha}\right)$ then the $\alpha$ coordinate of $x_{t}$ is $\left(x_{\alpha}\right)_{t}$. We make constant use of the following theorem due to Ellis [6].

THEOREM 4.2. If $(X, T)$ is a flow and if each orbit has compact closure then the following statements are equivalent:

(a) The flow $(X, T)$ is distal.

(b) For every cardinal a the flow $\left(X^{a}, T\right)$ is pointwise almost periodic. $\left(X^{a}\right.$ is the cartesian product of $X$ taken a times.)

(c) $(X \times X, T)$ is pointwise almost periodic.

Definition 4.3. We let $A(d)$ be the set of all functions which come from compact distal flows. 
We see easily that $A(d) \subset A(M)$ since the orbit closure of an almost periodic orbit is a minimal set.

THEOREM 4.4. $A(d)$ is a lattice algebra of functions.

Proof. It is clear that $A(d)$ is closed under scalar multiplication. Our result will follow immediately if we can prove the following statement: Let $x_{1}$ and $x_{2} \in A(d)$ and let $h: T \times T \rightarrow T$ be continuous in both variables then $h\left(x_{1}(t), x_{2}(t)\right)$ is in $A(d)$.

To prove this, we let $\left(X_{i}, T\right)$ be two compact distal flows and let $f_{i}: X_{i} \rightarrow T$ and $p_{i} \in X_{i}$ for which $x_{i}(t)=f_{i}\left(\left(p_{i}\right)_{t}\right)$. The flow $\left(X_{1} \times X_{2}, T\right)$ is compact distal. We define $g: X_{1} \times X_{2} \rightarrow T$ as follows $g\left(q_{1}, q_{2}\right)=h\left(f_{1}\left(q_{1}\right), f_{2}\left(q_{2}\right)\right)$. We see that $h\left(x_{1}(t), x_{2}(t)\right)=h\left(f_{1}\left(\left(p_{1}\right)_{t}\right), f_{2}\left(\left(p_{2}\right)_{t}\right)\right)=g\left(\left(p_{1}\right)_{t},\left(p_{2}\right)_{t}\right)$. This completes the theorem.

We remark that we have proven more than the theorem states. We have shown that any continuous combination of functions in $A(d)$ is again in $A(d)$. Since $A(M)$ is not an algebra we observe that the inclusion $A(M) \supset A(d)$ is proper. The next problem is to see if we can find a universal flow with which we can characterize $A(d)$.

Definition 4.5. A compact distal minimal flow $(D, T)$ is called a universal compact distal minimal flow if for any other compact distal minimal flow $(X, T)$ there is a homomorphism of $D$ onto $X$.

THEOREM 4.6. There is a unique universal distal minimal flow $(D, T)$.

Proof. We may prove the existence and uniqueness of $(D, T)$ in the same manner as Chu [4] proves the existence and uniqueness of the universal minimal set. We give here, however, a shorter proof suggested by Kakutani which takes advantage of the distal property. Let $\left(X_{\alpha}, T\right), \alpha \in A$, be the collection of all compact distal minimal flows. This leads to no contradiction since the cardinality of $X_{\alpha}$ is bounded by a fixed cardinal. For each $\alpha \in A$ and $p \in X_{\alpha}$ we let $X_{\alpha, p}=X_{\alpha}$. The flow $\left(\Pi_{\alpha, p} X_{\alpha, p}, T\right)$ is compact distal. The point $q \in \Pi_{\alpha, p} X_{\alpha, p}$ whose $\alpha, p$ th coordinate is $p$, is almost periodic and thus determines a minimal set $D$. It is easy to see that $(D, T)$ is universal. In fact $D$ contains a point $q$ such that if $(X, T)$ is a compact distal minimal flow and $p \in X$ then the homomorphism $\pi$ may be so chosen that $\pi(q)=p$. We call $q$ a distinguished point of $D$.

Suppose $(X, T)$ is another compact universal distal minimal set. There is a homomorphism $\psi$ of $X$ onto $D$. Let $p \in \psi^{-1}(q)$ and choose $\pi$ a homomorphism of $D$ onto $X$ such that $\pi(p)=q$. Since $\psi \circ \pi$ is the identity on the orbit of $p$ it is the identity on $D$. Thus $\pi$ is one-to-one and is a homeomorphism. Consequently $(D, T)$ and $(X, T)$ are isomorphic.

THEOREM 4.7. Let $(D, T)$ be the universal compact distal minimal flow. If $p$ and $r \in D$ then there is an isomorphism $\pi$ of $(D, T)$ onto itself such that $\pi(p)=r$.

Proof. Here we make use of the results of Ellis [5]. Let $E(D, T)$ be the enveloping semi-group of $(D, T)$. We first observe that the flow $(E, T)$ is distal. 
Suppose $\left\{t_{n}, n \in N\right\}$ is a net in $T$ and $f, g, h$ are in $E$ such that $t_{n}(f) \rightarrow h$ and $t_{n}(g) \rightarrow h$. This means for every point $u$ in $D$ we have $t_{n}(f(u)) \rightarrow h(u)$ and $t_{n}(g(u)) \rightarrow h(u)$. Since $(D, T)$ is distal we see that $f(u)=g(u)$ for each $u \in D$ and thus $f=g$.

Let $q$ be a distinguished point of $D$. Clearly it suffices to show that for each $p \in D$ there is an isomorphism $\pi$ of $(D, T)$ onto itself such that $\pi(q)=p$. Let $I$ be a minimal left ideal in $E(D, T)$. In [6] it is noted that $(I, T)$ is a minimal set and since $(E, T)$ is distal so is $(I, T)$. Let $\psi: I \rightarrow D$ be defined by $\psi(f)=f(q)$. The map $\psi$ is a homomorphism of $(I, T)$ onto $(D, T)$. Let $\pi$ be a homomorphism of $(D, T)$ onto itself such that $\pi(q)=p$. Since $(D, T)$ is universal distal and $(I, T)$ is distal there is a homomorphism $\phi$ of $(D, T)$ onto $(I, T)$. The composition $\phi \circ \pi \circ \psi$ is a homomorphism of $(I, T)$ onto itself and is thus an isomorphism [5, Lemma 5, p. 279]. It follows that $\pi$ is an isomorphism.

Definition 4.8. We let $A(D)$ be the set of all functions which come from the universal distal minimal flow.

THEOREM 4.9. $A(D)=A(d)$.

Proof. We clearly have $A(D) \subset A(d)$. The proof that $A(d) \subset A(D)$ is the same as that given in Lemma 3.6.

In $\S 3$ we were able to say that $x$ was in $A(M)$ if and only if its orbit closure in $(C, T)$ was a compact, pointwise almost periodic invariant set. That is, we could characterize $A(M)$ by properties of the flow $(C, T)$. The next theorem does this for $A(D)$.

THEOREM 4.10. The function $x$ is in $A(D)$ if and only if its orbit closure in $(C, T)$ is a compact distal minimal set.

Proof. Let $X$ be the orbit closure of $x$ in $(C, T)$. Suppose $(X, T)$ is a compact distal minimal flow. Define $f: X \rightarrow T$ as follows $f(y)=y(0)$. We see that $f$ is continuous and $x(t)=x_{t}(0)=f\left(x_{t}\right)$ so $x \in A(D)$.

Suppose now that $x \in A(D)$. There is a function $f: D \rightarrow T$ and $p \in D$ such that $f\left(p_{t}\right)=x(t)$. Let $\psi: D \rightarrow X$ be defined as follows $(\psi(q))(t)=f\left(q_{t}\right)$ for $q \in D$ and $t \in T . \psi$ is a continuous homomorphism of $(D, T)$ onto $(X, T)$. If we can show that $(X \times X, T)$ is pointwise almost periodic then the result would follow from Theorem 4.2( $\left.{ }^{3}\right)$. If $V$ is a neighborhood of $y$ in $X \times X$ and $q \in D \times D$ for which $\psi \times \psi(q)=y$ then there is a neighborhood $W$ of $q$ such that $\psi \times \psi(W) \subset V$. Since $(D, T)$ is distal it follows that $(D \times D, T)$ is pointwise almost periodic. There is a relatively dense set $S \subset T$ such that $q_{s} \in W$ for $s \in S$. Thus $y_{s}=$ $(\psi \times \psi(q))_{s}=\psi \times \psi\left(q_{s}\right) \in V$ for $s \in S$ and the theorem is completed.

In proving Theorem 4.4 we made use of the fact that if $(X, T)$ was a compact

(3) The remaining part of the proof is essentially a reiteration of Lemma 2(4), Ellis and Gottschalk, Homomorphisms of transformation groups, Trans. Amer. Math. Soc. 94(1960), 258-271. 
distal flow then the product flow $(X \times X, T)$ is pointwise almost periodic. The fact that the converse holds gives us the following theorem.

TheOREM 4.11. Let $A \subset A(M)$ and let $A$ be invariant under translation. Let $A$ contain the orbit closures of any points in it. If $(A \times A, T)$ is pointwise almost periodic then $A \subset A(D)$.

Proof. Since $A \subset A(M)$ it follows that the orbit closure in $(C, T)$ of each point of $A$ is compact. Since $(A \times A, T)$ is pointwise almost periodic it follows from 4.2 that $(A, T)$ is distal. Define $f: A \rightarrow T$ as follows $f(y)=y(0)$. If $x \in A$ then $x(t)=f\left(x_{t}\right)$ and we see that $x$ comes from the compact distal minimal flow defined on the orbit closure of $x$ in $(C, T)$ and the function $f$ restricted to this flow. The proof is completed.

We remark here that the usual method of proving that the uniformly almost periodic functions form an algebra is to show that two such functions have a common relatively dense set of translation numbers [3]. This amounts to showing that the product flow, induced by translation on uniformly almost periodic functions, is pointwise almost periodic. The previous theorem tells us essentially what restriction is put on a family of functions if every pair of its members has a common relatively dense set of translation numbers.

\section{Functions which come from equi-continuous transformation groups.}

Definition 5.1. If $(X, T)$ is a flow and $X$ is a uniform space then we say that that the flow is equi-continuous if the set of functions $\pi^{t}: X \rightarrow X$ (defined by $\left.\pi^{t}(x)=x_{t}\right)$ is an equi-continuous set.

Definition 5.2. We let $A(e)$ be the set of all functions $x \in C$ which come from a compact equi-continuous minimal flow.

Kakutani and Baum [2] have shown that $A(e)$ is contained in the collection of uniformly almost periodic functions. (Also see $[8,4.67]$.) In $(B, T)$ each uniformly almost periodic function $x$ has an orbit closure $X$ which is compact. In $(B, T)$ we see that $T$ acts as a group of isometries and thus $(X, T)$ is an equi-continuous minimal flow. The mapping $f: X \rightarrow T$ given by $f(y)=y(0)$ shows us that $x$ comes from the equi-continuous minimal flow $(X, T)$. We have proved

THEOREM 5.3. $A(e)$ is the set of uniformly almost periodic functions.

Chu [4] has shown that there is a unique compact universal equi-continuous flow $(E, T)$ and that $E$ is the Bohr compactification of the reals. If $A(E)$ is the set of all functions coming from the flow $(E, T)$ then we have the following:

THEOREM 5.4. $A(E)=A(e)$.

Since each function $x$ in $A(E)$ is uniformly almost periodic we see that its closure in uniform norm topology is compact. Thus its orbit closure in the compact open topology is the same as its orbit closure in the uniform norm topol- 
ogy and the two topologies are equivalent on the orbit closure. We thus have the following theorem.

THEOREM 5.5. The function $x \in A(E)$ if and only if its orbit closure $X$ in $(C, T)$ is compact and $(X, T)$ is an equi-continuous flow.

Kakutani's theorem [2] says that if all the functions which come from a minimal flow $(Y, T)$, on a compact metric space $Y$, are uniformly almost periodic then the flow $(Y, T)$ is equi-continuous. The authors along with L. Markus [1] have shown the existence of distal minimal flows $(Y, T), Y$ compact metric, which are not equi-continuous. Thus there is a function $x$ which is not uniformly almost periodic and which comes from a distal minimal flow. We thus have

THEOREM 5.6. $A(D) \neq A(E)$.

We owe to the referee the following remark, the observations $[\$ \S 3,4,5]$ carry over with little or no change to more general transformation groups and to functions which have values in a uniform space.

Added in proof. H. Furstenberg has pointed out that there is an example of a function in $A(D)$ which has no mean value. This example may be found on p. 584. H. Furstenberg, Strict ergodicity and transformations of the torus, Amer. J. Math. 83 (1961), 573-601.

\section{BIBLIOGRAPHY}

1. L. Auslander, F. Hahn and L. Markus, Topological dynamics on nilmanifolds, Bull. Amer. Math. Soc. 67 (1961), 298-299.

2. J. D. Baum, An equicontinuity condition for transformation groups, Proc. Amer. Math. Soc. 4 (1953), 656-662.

3. A. Besicovitch, Almost periodic functions, Cambridge Univ. Press, Cambridge, 1932.

4. H. Chu, On universal transformation groups, Illinois J. Math. 6 (1962), 317-326.

5. R. Ellis, A semigroup associated with a transformation group, Trans. Amer. Math. Soc. 94 (1960), 272-281.

6. — Distal transformation groups, Pacific J. Math. 8 (1958), 401-405.

7. F. Eberlein, Abstract ergodic theorems and weak almost periodic functions, Trans. Amer. Math. Soc. 67 (1949), 217-240.

8. W. Gottschalk and G. Hedlund, Topological dynamics, Amer. Math. Soc. Colloq. Publ. Vol. 36, Amer. Math. Soc., Providence, R. I., 1955.

9. M. Morse, Recurrent geodesics on a surface of negative curvature, Trans. Amer. Math. Soc. 22 (1921), 33-51.

10. V. V. Nemyskii, Topological problems of the theory of dynamical systems, Amer. Math. Soc. Transl. No. 103 (1954), 15-889.

11. J. Oxtoby, Ergodic sets, Bull. Amer. Math. Soc. 58 (1952), 116-136.

12. A. Weil, L'intégration dans les groupes topologiques et ses applications, Hermann, Paris, 1940.

\footnotetext{
INDIANA UNIVERSITY, BLOOMINGTON, INDIANA

YALE UNIVERSITY, New HAVEN, ConNecticut
} 